\title{
Las estrategias de implicación emocional en anuncios en prensa escrita alemana
}

\author{
Marta PANADÉS GUERRERO \\ Universitat de Barcelona \\ martapanades@ub.edu
}

Recibido: 28 de noviembre de 2013

Aceptado: 21 de febrero de 2014

\section{RESUMEN}

Dada la importancia de las emociones en la toma de decisiones humanas señalada en los últimos años desde la denominada inteligencia emocional, el objetivo de este artículo se ha centrado en comprobar cómo se manifiestan los aspectos emocionales en un nivel formal en una comunicación básicamente persuasiva como es la publicitaria, y más concretamente en el caso de los anuncios en prensa escrita alemana. Para ello, se ha realizado un análisis cualitativo donde se han codificado los indicadores encontrados a este respecto, seleccionados tanto a partir de categorías existentes en la literatura como de los datos emergentes del corpus. Posteriormente se ha clasificado el catálogo de categorías resultantes según el vínculo existente entre los interlocutores (proximidad/distancia) o el uso de la empatía. De este modo, la confirmación de la presencia de la emocionalidad en los anuncios publicitarios ha reflejado atributos de una sociedad contemporánea enfocada hacia el divertimento y la búsqueda de emociones.

Palabras clave: Textos publicitarios en alemán, emocionalidad, estrategias persuasivas, cortesía, estrategias indirectas.

\section{Emotional Strategies in Advertisements in the German Press}

\begin{abstract}
Due to the increasing importance of emotionality in human decision-making pointed out in recent years from the emotional intelligence, this paper focuses on the presence of emotional aspects in a formal level in persuasive oriented texts such as advertising texts, and in particular in advertising texts in the German press. Thus, it has been carried out a qualitative analysis in order to codify indicators regarding emotionality. Those have been selected both from existing categories in the literature, as well from own developed categories. At that end, the final list has been classified according to either the existing bound between participants (proximity/distance) or to the use of empathy. Therefore, it can be argued that the presence of emotional aspects reflects indisputable characteristics of a contemporary society focused on leisure and the emotional search.
\end{abstract}


Keywords: Advertising Texts in German, Emotionality, Persuasive Strategies, Politeness, Indirectness.

RESUMEN: 1. Introducción. 2. La dimensión afectivo-emocional. 3. Procedimiento. 3.1. Estrategia metodológica. 3.2. Corpus. 4. Análisis cualitativo y codificación de los indicadores. 4.1. Las estrategias de proximidad y distancia y creación de vínculo entre los interlocutores. 4.2. Las estrategias de empatía. 5. Conclusiones.

\section{Introducción}

La hipótesis inicial de la que parte el presente artículo plantea comprobar cómo se manifiesta la emocionalidad en la parte verbal del texto en los anuncios publicitarios en prensa escrita alemana. Uno de los aspectos más estudiados de este tipo textual desde la perspectiva lingüística ha sido la relación entre la imagen y la parte verbal, así como las funciones desempeñadas por cada una de ellas ( $c f$. entre otros, Sowinski 1998: 16; Nöth 2000: 489-496; Stöckl 2004; Schmitt 2008; Hamilton 2009; García-Velasco 2011; Lagerwerf 2012; Rentel 2011). Tradicionalmente, la primera se había fijado como portadora de evocación y emocionalidad, y deja para la parte verbal la función más racional o informativa. De esta manera, el lenguaje verbal ha sido considerado el depositario de las funciones informativas del texto publicitario, esto es, donde se suelen especificar las atribuciones concretas de las características y apariencia de un producto, tanto mediante argumentos lógico-formales como racionales basados en datos o probabilidades, mientras que el estudio de la parte icónica se ha centrado en su función de atracción y activación de la atención del destinatario ${ }^{1}$.

Sin embargo, cada vez más las últimas investigaciones están ofreciendo una perspectiva diferente. Janich, además de señalar la prioridad de las imágenes en la realización de los spots publicitarios, examina el uso de los aspectos técnicos, concluyendo que la publicidad no tiene una función informativa: "Information in der Werbung gibt es also in eingeschränktem Mass [...], sie ist aber immer ausgesprochen selektiv und zweckorientiert und oft kombiniert mit nur scheinbar informativen Angaben" (Janich 1998: 38).

1 A pesar de que la oposición absoluta entre los conceptos de información y emocionalidad ya fue apuntada como errónea por algunos autores (RÖMER 1968), la tendencia ha sido considerar la publicidad como portadora de información y entender la parte emocional como parte de la irracionalidad. Esta misma autora afirma además que la parte verbal tiene un papel dominante frente a la imagen, puesto que la influencia en comunicación publicitaria solamente puede ser efectiva a través de la palabra, limitando la segunda a una función meramente de estimulación. En estudios posteriores se mantuvo un tratamiento similar, bien relegando la imagen a un rol secundario cuyo objetivo primordial es captar la atención de consumidor y presentando la parte verbal como el vehículo de información más importante (BAUMGART 1992); bien considerando la función de la emocionalidad como el hilo conductor entre afirmaciones y sensaciones para provocar reacciones a partir de asociaciones, relatos cercanos y sentimientos de proximidad (MOSER 2002). En esta misma línea, SOWINSKI (1998: 31) distingue la información como un aspecto fundamental en la comunicación publicitaria, puesto que da a conocer los fines o características de un producto cuya finalidad es incitar a la adquisición de estos. 
Por otra parte, Hennecke (1999: 23) señala la tendencia existente hacia el divertimento y el individualismo en las estrategias y formas de comunicación contemporáneas, hecho que implica una concepción de la sociedad enfocada al ocio, tal y como es la sociedad en la que se desarrollan los anuncios objeto de esta investigación. Desde una dimensión pragmático-funcional, esta autora ha analizado las diferencias del efecto emocional en la producción y recepción de un mismo mensaje publicitario para consumidores del antiguo Este y Oeste de Alemania. Teniendo en cuenta que el entorno social y económico configura un marco donde las estrategias publicitarias se adaptan a los diferentes grupos sociales acorde a sus expectativas, creencias y valores, su estudio confirma que en el caso de la antigua RDA se desarrolló una publicidad de carácter más informativo, muy diferente de las estrategias persuasivas utilizadas en la antigua RFA, enmarcada en una economía de mercado y con una sociedad enfocada al consumo y al tiempo libre. Del mismo modo, Lacroix (2005) interpreta sociológicamente el papel de la emocionalidad en la comunicación publicitaria, afirmando que su función es llenar un vacío social motivado por la falta de proyectos, y que su uso reiterado se debe al estímulo constante que representa para el destinatario. Asimismo, Ungerer (2004: 307-328) ha estudiado la relación entre anuncios publicitarios y noticias de prensa escrita en lengua inglesa, poniendo de relieve por un lado el deseo, y por tanto un aspecto emocional, como fuerza motora que induce a la adquisición del producto anunciado y, por otro, la utilización del tipo textual noticia para otorgarle credibilidad.

En cuanto a los estudios desde la psicología del consumo, a partir de los años ochenta las investigaciones empezaron a centrarse en las reacciones emocionales del consumidor y a estudiar el papel de los sentimientos y las emociones en la efectividad de los anuncios publicitarios, confirmando la importancia y variedad de la relación emoción-publicidad, fundamental a la vez que compleja (Edell 1990: XIV; Puig i Borràs 2010: 34-39).

Igualmente, son numerosos los estudios empíricos en comunicación publicitaria. Dmoch (1997) ha analizado el papel de las emociones en publicidad en lengua alemana, tanto en su aspecto visual como verbal y paralingüístico, corroborando el papel de la emocionalidad en la descripción de las características de un producto, y apuntando a una preferencia en el uso de las vivencias emocionales (emotionale Erlebnisse, Dmoch 1997: 27) con el fin de provocar en el destinatario asociaciones entre la marca y una experiencia personal ${ }^{2}$. Del mismo modo, se ha puesto de relevancia un cambio de valores en la presentación del mensaje publicitario (Dmoch 1997; Kroeber-Riel, 2000), tomando como ejemplo productos como los del sector financiero o sanitario, que anteriormente basaban su estrategia en datos específicos y lógicos, y que ya están utilizando una estrategia más emocional y empírica ante mercados saturados con mayor oferta que demanda.

${ }^{2}$ Los contenidos desde una perspectiva emocional también contemplan la inclusión de sentimientos, evocaciones, vivencias o experiencias que apelen directamente al destinatario y permitan el desarrollo de un patrón identificativo enunciador-destinatario. 
Por tanto, los trabajos sobre los aspectos evocadores y connotativos en el uso de las emociones han empezado a tener así un papel predominante, pasando de ser referente secundario a núcleo central de la estrategia comunicativa en publicidad (Messaris 1997: 34). De forma paralela al desgaste que se produce en sus formas de comunicación, se incrementa la utilización de nuevas fórmulas estratégicas donde la emocionalidad tiene un papel decisivo, denominándose incluso como posición tradicionalista la manera de entender la estrategia publicitaria desde la transmisión de información (Kroeber-Riel 1994). De este modo, la tendencia actual parece decantarse por un aumento en el uso de la emocionalidad como argumento directo (Jiménez Morales 2006: 118ss.; Larminaux 2010: 438), aunque sin perder de vista que necesita elementos complementarios que acaben de redondear el mensaje publicitario.

Todos estos elementos apuntan hacia una misma dirección, la de la cada vez mayor relevancia social de la parte emocional en múltiples aspectos de la vida humana, desde la toma de decisiones en conductas cotidianas hasta la elaboración de estrategias comunicativas, enlazando así con las aportaciones de los últimos años, enmarcadas dentro de la llamada inteligencia emocional, que establecen una relación entre razón y emoción, entre conceptos codificados en entradas léxicas, estructuras gramaticales y modelos mentales de acción e interacción, y priorizan la importancia de las emociones en la toma de decisiones humanas ( $c f$. entre otros Gigerenzer 2000; Damásio 2006; Ariely 2008). Por tanto, parece pertinente preguntarse cómo afecta este fenómeno a la formulación de la parte verbal del anuncio publicitario, la cual a pesar de verse paulatinamente reducida (Wehner 1996: 147; Dirscherl 1999), continúa siendo un elemento constitutivo inherente. Por todo esto, el objetivo del análisis se centrará en determinar las estrategias persuasivas de implicación emocional en la parte verbal del texto, identificando la presencia de recursos lingüísticos portadores de aspectos evocativos y connotativos.

\section{La dimensión afectivo-emocional}

Una vez concretado el objetivo que se va a investigar en los recursos de expresión de la emocionalidad, o lo que es lo mismo, en las estrategias persuasivas de implicación emocional, la definición "geprägter Terminus zur Bezeichnung sprachlicher Mittel, die dazu verwendet werden, Emotionen auszudrücken, z. B. Ausruf, Frage, Wunsch, Befehlssatz..." (Bussmann 1990: 208) servirá para acotar su significado, presentando el adjetivo emotivo en relación con la parte verbal del discurso. Asimismo, desde el marco de los estudios de semántica y pragmática intercultural, Wierzbicka (1996: 142) diferencia entre sensación (sentimiento físico de causa fisiológica) y emoción (sentimiento mental libre del control de la voluntad y consecuencia de pensamientos y actitudes). Además, De Beaugrande y Dressler, aplicando este concepto a la textología por primera vez, incluyen la experiencia vivencial dentro del concepto de emocionalidad cuando apuntan a "un estado experiencial y no neutral con respecto a una entidad creada sensorialmente" (De Beaugrande / Dressler 1997: 150), hecho que remite a la memoria episódica de la lingüística cog- 
nitiva $^{3}$. En esta misma línea, Peña Cervel (2003: 320), desde una aproximación cognitiva a las imágenes-modelo, reafirma el componente vivencial como fundamental para la conceptualización de emociones en lengua inglesa. En consecuencia, la argumentación persuasiva dentro del contexto de la comunicación publicitaria también apelará a la emoción, evocando sentimientos, sensaciones o experiencias subjetivas. Es decir, la emoción no procederá de las características intrínsecas del producto, sino de la activación de esquemas mentales de un mundo ideal al que se accede mediante el producto.

En el caso que nos ocupa, la emocionalidad se define por tanto como un estado que reactiva la experiencia vivencial del sujeto, evocada por recursos lingüísticos aparecidos en el texto. Aquí hay que recordar igualmente que las imágenes también desempeñan un papel importante, aunque para este estudio solamente se tendrán en cuenta si tienen una relación de interdependencia con las estrategias verbales que aparezcan en el texto. Todo esto no hace sino reflejar la aproximación interdisciplinar que necesita el tema tratado (desde la lingüística y las ciencias sociales), donde el hilo conductor es la comunicación textual, así como su actualidad e importancia. A este respecto, cabe mencionar que en los últimos años se han realizado tanto investigaciones desde la lingüística interdisciplinar sobre el uso del lenguaje aplicado en un contexto determinado (Tienken 2008; Larminaux 2010), como trabajos sobre el nexo entre emociones y publicidad desde la psicología y la comunicación (Jiménez Morales 2006; Puig i Borràs 2010).

\section{Diseño metodológico}

Teniendo en cuenta el objetivo específico mencionado anteriormente, a continuación se describirá brevemente la estrategia metodológica utilizada para su análisis, mientras que en el apartado siguiente (3.2) se detallará el procedimiento de selección y tratamiento del corpus.

\subsection{Estrategia metodológica}

Para la selección de las estrategias de implicación emocional se han tenido en cuenta tanto categorías señaladas en la literatura como categorías de elaboración propia emergentes de los datos que conforman el corpus, hecho que ha permitido identificar y describir qué grado de influencia tienen en la formulación de los anuncios en prensa escrita.

Con el fin de facilitar el análisis, el corpus se ha recopilado en formato papel y se han digitalizado los textos-anuncio originales. A continuación se ha transcrito la parte verbal de cada uno de ellos, disociándola de este modo de la parte icónica, y se han presentado los datos para que pudieran ser codificados posteriormente mediante el

${ }^{3}$ Entendida como aquel tipo de conocimiento de parámetros espacio-temporales y codificación explícita correspondiente a un episodio o vivencia compartidos, y que tiene como función localizar una situación determinada en un episodio mental específico previamente almacenado. 
programa NVivo. De esta manera, el corpus ha quedado además preparado como material didáctico para ser utilizado en el aula de alemán para usos específicos. A continuación, se ha realizado la codificación de los recursos lingüísticos en un nivel semántico-proposicional (incluyendo tanto oraciones como grupos nominales), y se han clasificado en dos grandes grupos: establecimiento de vínculo a través de la proximidad o la distancia entre los interlocutores, y análisis de la empatía.

El programa de análisis cualitativo de datos utilizado (NVivo) ha posibilitado de forma paralela un análisis tanto inductivo como deductivo de los indicadores textuales. Las categorías abiertas se han ido creando a medida que avanzaba el análisis, y se han ido incorporando como categorías libres (free nodes) para posteriormente ser incluidas dentro de la clasificación jerarquizada de las categorías cerradas (tree root), pudiéndose así estructurar los datos y establecer las interrelaciones posteriores necesarias. La identificación de categorías cerradas basadas en piezas léxicas se ha realizado de manera automática mediante la función de búsqueda textual (text search), que detecta las unidades seleccionadas y permite incorporarlas más adelante a la categoría deseada. Así, el programa permite combinar ambos tipos de análisis: los free nodes sirven como un almacén de categorías nuevas resultantes de un análisis inductivo, mientras que a partir del tree root se pueden jerarquizar y ordenar las categorías preestablecidas, incluidas las categorías abiertas. Del mismo modo, mediante la función index search se han podido tanto sumar los resultados de las diferentes categorías o subcategorías (función union), como crear interrelaciones entre ellas según los parámetros deseados (función intersection).

\subsection{Corpus}

Para llevar a cabo este estudio se ha creado un corpus lingüístico de anuncios gráficos de prensa escrita procedente de revistas de difusión masiva en Alemania. Para garantizar un mínimo de homogeneidad y representatividad, se ha optado por seleccionar los anuncios acotados por un periodo temporal hasta llegar a un número significativo (en este caso, los publicados entre enero de 2000 y diciembre de 2003). En concreto, los anuncios proceden mayoritariamente de los semanarios Stern (de octubre de 2001 a octubre de 2003); Spiegel (de enero de 2000 a julio de 2002) y Focus (de enero de 2000 a diciembre de 2001), y se ajustan de este modo a los criterios de variedad y diversidad necesarios.

Además, se han adoptado dos restricciones iniciales: la primera, de tipo temático, determinada por la selección de los anuncios de entre la denominada publicidad comercial (cf. Wirtschaftswerbung 4 , Janich / Greule 2001: 20). En ningún caso se han contemplado los anuncios de tipo industrial y de prensa especializada debido a su sesgo, ni los anuncios clasificados por considerarse que no existe inicialmente una voluntad persuasiva ni una utilización de estrategias indirectas por parte del enunciador. La segunda restricción se debe al hecho de no haber valorado la posi-

\footnotetext{
${ }_{4}^{4}$ Tipo de publicidad donde queda excluida la propaganda política, la publicidad cultural o religiosa y la publicidad directa, hecho que propicia una aproximación del lenguaje y las estrategias utilizadas a las tendencias que se producen en la sociedad (CoOK 1992: 16s.).
} 
ción de los anuncios dentro de una campaña determinada (de lanzamiento, perpetuación de la imagen, campaña de nuevo producto, campaña de precio etc.), puesto que en su selección no se ha realizado un seguimiento concreto de cada producto. Dados los objetivos de la investigación, se han descartado los anuncios con una función subsidiaria del texto y se han elegido los anuncios con textos más extensos (high-involvement Anzeige (Janich / Greule 2001: 25)). No obstante, la imagen ha sido considerada un referente para el análisis cualitativo por ser indicativa de formas especiales de coherencia en la relación parte verbal/parte visual (Möhn 2000: 565).

Atendiendo a estos criterios de selección, el corpus se compone de un total de 174 textos-anuncio extraídos de las fuentes anteriormente mencionadas, referentes a productos desconocidos para la investigadora con el fin de propiciar un distanciamiento y evitar asociaciones que pudieran distorsionar el análisis, siendo el número final de palabras de 18.037, con un valor medio de 104 palabras por texto-anuncio.

\section{Análisis cualitativo y codificación de los indicadores}

Una vez concretado el concepto de emocionalidad en el apartado 2, es necesario determinar sus recursos lingüísticos de expresión en alemán aparecidos en los textos-anuncio. A continuación se comentarán brevemente los indicadores hallados y se mostrará un ejemplo de cómo se ha llevado a cabo el análisis cualitativo, para más adelante (4.1 y 4.2) explicar en más profundidad las estrategias persuasivas de implicación emocional.

En este punto, cabe mencionar las estrategias de cortesía positiva recogidas por Brown / Levinson (1987: 101ss.), que inciden en aquellos aspectos que acentúan el vínculo entre los interlocutores con el fin de acercar la intención del enunciador a las expectativas del destinatario. Según su función se han clasificado en los dos grupos que se detallan a continuación:

1. Las "estrategias de proximidad y distancia y creación de vínculo entre los interlocutores" estrechan el vínculo entre estos, tanto acercando la intención del enunciador a las expectativas del destinatario e implicándolo emocionalmente, como confirmando su identidad social. Algunos indicadores se han adaptado a las particularidades de la lengua alemana, como son las diferentes formas de presentación de los interlocutores (use of (non)-honorific pronouns, the inclusive we form) en un nivel gramático-léxico. Como indicadores discursivos se han seleccionado categorías como el discurso directo, el uso estratégico del humor y de la ironía, el registro (disglosia, jargon or slang) y la recreación de otros tipos textuales (joke, gossip, small talk) en el sentido de géneros (Textsorten), que en el mensaje publicitario se utilizan persuasivamente como mecanismos de una "indirección argumentativa" (Larminaux 2010: 484).

2. En cuanto a "las estrategias de empatía", se han seleccionado aquellas categorías que permitan realizar asociaciones que evoquen directamente esquemas emocionales o comporten una carga connotativa relevante, como el uso del presente (vivid present), las partículas modales en combinación con las 
formas exhortativas (softening the imperative); los operadores emotivos, evaluativos e hipotéticos (intensifying modifiers) y la inclusión de una narración (story telling) que implique directamente al destinatario. En este punto cabe recordar de nuevo que es necesario adaptar el modelo universal propuesto por Brown / Levinson (1987: 101ss.) al caso concreto de la lengua y cultura alemanas (cf. Siebold 2000; Eelen 2001; House 2005: 13-28), que poseen manifestaciones y características propias.

Debido a que en la comunicación publicitaria existe una búsqueda constante de motivos visuales que apelen al imaginario emocional del destinatario, dentro de este segundo grupo también se han tenido en cuenta las imágenes-modelo (Schema-Bilder) propuestas por Tepe / Zur Nieden (2001: 32ss.). De esta forma, tal y como señala Puig i Borràs (2010: 63), el productor de un anuncio publicitario (en el sentido de conceptualizer y formulator a la vez de Levelt 1989: 2ss.) necesita comprender y conocer las necesidades y deseos de su público objetivo (target) con el fin de generar motivaciones y conseguir de este modo llevar a la realización de una conducta determinada, en este caso la adquisición del producto. Respecto a la creación de un marco que pueda servir para clasificar los aspectos emocionales aparecidos en los textos publicitarios, Kroeber-Riel (2000: 27) presenta una serie de escenas conceptuales, mientras que Dmoch (1997: 87ss.) se decanta por una tipología de los lugares comunes según su área de influencia: los universales o éticos, independientes de experiencias individuales; los culturales y los específicamente locales o grupales. De este modo, partiendo de unas categorías abiertas y sin una jerarquía previamente establecida, las propuestas de estos autores serán de utilidad como punto de partida para la clasificación general del plano semántico-proposicional.

"Ich bin ein Sächsist".

Immer mehr Menschen bekennen sich zum Sächsismus. Kein Wunder, denn was Sachsen zu bieten hat, entfacht Leidenschaften: die faszinierende Kombination aus Kultur \& Natur, Städten \& Landschaften abseits ausgetretener Pfade. Besuchen Sie die Musik-Stadt Leipzig - hier spielt die Musik, 2 x Übernachtung/Frühstück im Hotel Marriott - first class ab 77,00€ p. P. inkl. einer Leipzig-Card (Tagescard) mit freier Fahrt auf allen Linien der LVB und jede Menge Preisvorteile bei Stadtrundgängen, Museen, ausgewählten Konzerten etc. Nähere Infos und weitere Top-Angebote unter $0351 / 491700$ oder unter www.sachsen-tourismus.de. Wann werden Sie Sächsist?

(Stern 17/2003. Anunciante: Sachsen Tourismus).

Como muestra, en el texto-anuncio transcrito sobre estas líneas, se han encontrado indicadores como las referencias directas a los interlocutores y el discurso directo, mediante el que se promueve el interés del destinatario incluyéndolo como participante en un diálogo (Ich bin ein Sächsist; Wann werden Sie Sächsist? Das haben Sie noch nie gesehen). Por otro lado, el uso del humor y de la ironía permite crear un vínculo de proximidad entre los interlocutores por la existencia de un conocimiento compartido por ambos (Immer mehr Menschen bekennen sich zum Sächsismus; Wann werden Sie Sächsist?). Igualmente, como recurso gramático-léxico, el uso del adjetivo sirve como indicador de una perspectiva subjetiva (die faszinieren- 


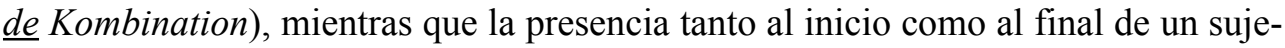
to vivencial (Ich bin ein Sächsist; Das haben Sie noch nie gesehen) se corresponde con una apelación directa al destinatario, donde el enunciador desea compartir experiencias y valores recurriendo a un lugar común ( $c f$. "claim common ground" de la teoría de la cortesía, Brown / Levinson 1987: 199ss.): no ser sexista. En este caso, el topoi se basa por un lado en una presunción falsa de valores no compartidos (nadie se declara sexista -sächsist- en un lenguaje políticamente correcto) utilizando el tipo textual reconocimiento de culpa (Geständnis); y por otro lado en una asunción falaz - ¿solo en Sachsen lo podrás encontrar?- de valores compartidos (Natur, Kultur, Landschaftten) a través de la reinterpretación forzada por la implicatura no conversacional (homofonía de sexist y sächsist). Por otra parte, la información semántico-proposicional referida a valores éticos y culturales (Landschaften abseits ausgetretener Pfaden) se ha codificado como "apelación a necesidades reales".

\subsection{Las estrategias de proximidad y distancia y creación de vínculo entre los interlocutores}

Bajo esta categoría se han identificado y clasificado aquellos recursos utilizados por el enunciador con la intención de acercarse al destinatario y simular una interacción con él, mientras que en el apartado siguiente (4.2) se ha codificado la intención de transmitir y despertar emociones no relacionadas directamente con el enunciador bajo la categoría "estrategias de empatía".

Se han encontrado recursos gramático-léxicos como la referencia directa a los interlocutores ( $c f$. Teilnehmer-Rollen de Levinson 2000: 74s.), manifestados mediante pronombres comunicativos con función deíctica, incluyendo tanto estrategias de proximidad como de distancia, así como referencias a la autoridad. En cuanto a las múltiples figuras del enunciador (Schwitalla 2001: 1.355ss.), se ha diferenciado entre el enunciador explícito en el texto en referencia al anunciante o creativo publicitario del producto (ejemplo 1) y el enunciador escenificado, incluido posteriormente en la categoría "sujeto vivencial" dentro de las estrategias de empatía. Para su identificación y codificación se han tenido en cuenta los pronombres personales y posesivos de primera persona del singular y/o del plural, y el uso de las comillas como marca de enunciación directa o monólogo interior. Tal y como puede verse en el ejemplo siguiente, los pronombres wir y unseren remiten a la empresa productora y no a un enunciador ficticio o escenificado, aunque sin perder de vista que la formulación ha sido realizada por el copy o creativo de la agencia publicitaria y no directamente por la empresa productora:

(1) Weihenstephan. Frischer Joghurt mild.

Heute verraten wir Ihnen das Rezept für unseren unnachahmlich cremigen, frischen Joghurt.

(Stern 44/2003. Anunciante: Weihenstephan) .

En cuanto a la presencia explícita del destinatario, se ha contemplado la distancia entre los interlocutores mediante el uso de pronombres de cortesía (Ihnen en el

\footnotetext{
${ }^{5}$ Las cursivas en los ejemplos son de la autora.
} 
ejemplo 1), que ponen de manifiesto por una parte las diferencias sociales relativas al rol de enunciador y destinatario, concretando así la identidad social de este último, y por otra crean un vínculo de reconocimiento al expresar respeto. También se han codificado como indicadores los pronombres personales y posesivos de segunda persona del plural y/o singular, incluyendo los de cortesía, y los emisores honoríficos y títulos codificados mediante piezas léxicas como Dame, Herr, Frau, Dr. etc. A continuación se presenta un ejemplo donde, además de la presencia de un enunciador escenificado (wir), también está presente la figura del destinatario, en este caso manifestado mediante los pronombres de cortesía Sie e Ihnen:

(2) Lieber Familienvater, natürlich möchten wir nicht, dass Ihnen etwas passiert. Dennoch wünschen wir Ihnen und Ihrer Familie, dass Sie im Fall einer plötzlichen Berufsunfähigkeit optimal versorgt sind. Auf Vater Staat können Sie dabei nicht mehr zählen.

(Stern 00/2003. Anunciante: Victoria Versicherungen).

También dentro de esta figura, para la codificación de la creación de proximidad se han buscado indicadores como los pronombres personales $d u / i h r$ y sus correspondientes pronombres posesivos. En el ejemplo siguiente (3), donde se escenifica la concepción intercultural de las relaciones mediterráneas, caracterizadas por la calidez social, el uso del tuteo y un entorno relajado, los interlocutores del texto se sitúan en el contexto mencionado utilizando las normas de comunicación pertinentes (dich, deine):

(3) Auf uralten Wegen wandern oder an einem Glas Morellino di scansano nippen, alles in den Terme die Saturnia ist bereit, dich zu neuem Leben zu erwecken. Eine Reise ins Herz der Toskanischen Maremma, in ein nie gekanntes Wohlbefinden, das deine Sinne belebt.

(Vogue Juli 2002. Anunciante: Terme di Saturnia).

Dentro de los recursos discursivos se ha tenido en cuenta el uso del discurso directo, utilizado tanto por parte del enunciador explícito en el texto (en el sentido de anunciante/creativo publicitario) como por el enunciador escenificado, para apelar al destinatario de forma directa y específica con la intención de que sea incluido como participante en el diálogo (Brown/Levinson 1987: 103-129). Por ser una categoría abierta, se ha codificado a partir del análisis de cada texto-anuncio, mientras que para su identificación no se ha tenido en cuenta la presencia de pronombres personales y posesivos (analizados anteriormente dentro de la categoría "referencia directa a los interlocutores"), sino el uso de las comillas como indicador de enunciación directa o monólogo interior, tal y como muestra el ejemplo 4:

(4) "Seit ich hier eingezogen bin, bin ich viel besser gestimmt. Das liegt wohl am Boden. Der trifft den passenden Holzton und knarrt niemals dazwischen. Zusammen sind wir das perfekte Duo".

(Stern 45/2002. Anunciante: Parador Holzböden).

Otro indicador de la perspectiva subjetiva es el uso del humor y de la ironía, que exigen un conocimiento compartido por los interlocutores y unas presuposiciones 
por parte del destinatario (Brown/Levinson 1987: 103-129). En este caso, ha sido necesario tener en cuenta la parte visual para su identificación, como en el caso del ejemplo siguiente (5), donde se hace referencia a un cuerpo de texto extenso realizado por el enunciador escenificado:

(5) Für alle, die sich mehr im Leben zu sagen haben als 160 Zeichen, gibt es jetzt imode, den multimedialen Begleiter, mit dem man bis zu 1.000 Zeichen über i-modeMail versenden und erhalten kann, immer und überall im E-Plus Netz.

(Stern 18/2002. Anunciante: e.plus).

Por otro lado, la recreación de tipos textuales activa escenarios de interpretación compartidos por los interlocutores (en el sentido de frames), formando un marco de interacción prototípico. De este modo, se pone de manifiesto una de las características más importantes del lenguaje publicitario: su carácter imitativo de otros tipos textuales ( $c f$. Larminaux 2010: 484 y el concepto de "intertextualidad" de De Beaugrande / Dressler 1997: IX, 249). Para ilustrar esta categoría, en el ejemplo siguiente (6) se reproduce la fórmula habitual de una felicitación y de un discurso de agradecimiento (Danksagung):

(6) Herzlichen Glückwunsch, Zukunftsfrau.

Jetzt würdest du ja sicher gerne deiner Mutter die tolle Neuigkeit erzählen. Aber wie? Mit deiner Persönlichen Kommunikations-Maschine (PKM) von Xelibri. Ganz einfach. Mach dich bei deiner Mutter bemerkbar, und zwar durch etwas, das wir "Anruf" nennen. Das Xelibri 3 schickt dann deine freudigen Worte zu ihr. Dabei verwendet es die Unterhaltung-durch-die-Luft-Technologie.

"Ich danke meiner Mutter, meinem Vatter, meinem Xelibri..."

(Stern 41/2003. Anunciante: Xelibri).

Al igual que la categoría anterior, el uso estratégico del registro (Brown/Levinson 1987: 103-129) se despliega en la polivalencia del lenguaje publicitario, que si bien pertenece al ámbito público, utiliza registros propios de esferas privadas o semiprivadas, pudiéndose utilizar para simular un diálogo determinado, donde el enunciador adquiere un rol y registros concretos para interpelar al destinatario con la intención de que este sea más proclive al producto o servicio. Para el análisis cualitativo se ha identificado y marcado el uso de registros propios del lenguaje coloquial (ejemplo 7), que posibilita la evocación de asociaciones y actitudes compartidas entre los interlocutores; el lenguaje juvenil y el uso del inglés por asociarse a valores como la actualidad, la contemporaneidad y la juventud (ejemplo 8); el baby talk y el lenguaje vulgar:

(7) Genau genommen: verdammt viele Kunden.

(Stern 27/2003. Anunciante: JET Tankstellen).

(8) HEY, UND WAS WIRD AUS MIR?

(Stern 27/2003. Anunciante: PKV).

Por otro lado, el uso de cultismos se ha entendido como una estrategia de auto$\mathrm{ridad} /$ distancia en oposición a los recursos de proximidad. Este tipo de registro 
incluye el lenguaje científico y académico (ejemplo 9) y el lenguaje poético (ejemplo 10):

(9) Mittags Currywurst - abends Bier und dann die eine oder andere Zigarette. Dann stauen sich die Stoffwechselgifte im Körper und die Leberwerte gehen hoch.

HepaBesch fördert die Regeneration belasteter Leberzellen und unterstützt den Leberstoffwechsel.

(Stern 26/2003. Anunciante: HepaBesch).

(10) DER MOND MALTE EIN BILD AUF MEINE HAUT. ER VERWANDELTE JEDEN QUADRATZENTIMETER IN EIN KUNSTWERK AUS UNSAGBAR WEICHEM LICHT.

(Stern 45/2002. Anunciante: Schiesser).

\subsection{Las estrategias de empatía}

Las categorías de este grupo se caracterizan por sus posibilidades de transferir o despertar emociones o sensaciones en el destinatario, que lo predisponen a que se identifique con el consumidor del producto anunciado. Tal y como ha sucedido en el grupo anterior, se han tenido en cuenta tanto recursos gramático-léxicos como discursivos.

Este tipo de estrategias se han identificado a partir del análisis de la valoración subjetiva mediante recursos gramático-léxicos como el tipo de predicado, que hace referencia a los verbos que expresan vivencias, emociones y sensaciones relacionadas con los objetos o acontecimientos referenciados o evocados o en usos metafóricos en este sentido (ejemplo 11), mientras que los adjetivos se han incluido por sus referencias a la perspectiva del enunciador respecto a la descripción o valoración subjetiva de unos hechos determinados (ejemplo 12):

(11) MEIN GARTEN - EIN ORT, AN DEM MEINE LEBENSLUST AUFBLÜHT. Jeder wünscht sich einen Ort, der anregt. An dem Körper und Geist Erholung finden und der Kraft und Energie spendet. Der eigene Garten ist so ein Ort. Er ist eine grüne Oase und ein kleines, privates Paradies. Und er ist genauso individuell wie Sie: $\mathrm{Ob}$ Sie von einem Steingarten träumen, sich einen Schwimmteich wünschen oder Ihre Lieblingsecke mit einer neuen Terrasse verschönern wollen.

(Brigitte, 15/2000. Anunciante: Garten \&Landschaft).

(12) Hier erwarten Sie die Farben der Saison: fröhliches Sonnengelb, himmlisches Azurblau, duftiges Grasgrün und vor allem blütenfrisches Rosa, kilometerweit: Mandelblüte, live und in der Farbe, zu der am besten ein rubinroter Pfälzer Spätburgunder passt. (Stern 10/2003. Anunciante: Deutsche Weinstrasse).

Por otro lado, las partículas (Helbig 1990b) (ejemplo 13) hacen referencia a las valoraciones y expectativas de los interlocutores respecto a las proposiciones expresadas, cambiando sus efectos de contenido según el contexto lingüístico o situacional. Para el análisis se han seleccionado las partículas modales (Abtönungspartikeln), las partículas gradativas (Gradpartikeln) y las partículas de contraste (Steigerungspartikeln), agrupadas posteriormente en una única categoría para evitar una elevada disgregación que provocara resultados sesgados: 
(13) Falls Sie mal ausser der Reihe die Abenteuerlust packt. Neu herausgegebene Papiere, mit Ausnahme von Anleihen und Schatzanweisungen, sogar ohne Gebüren. (Stern 44/2002. Bundesrepublik Deutschland Finanzagentur GmbH).

Por último, los operadores pragmáticos expresivo-valorativos (Calsamiglia/ Tuson 2004: 179ss.; Modalwörter de Helbig 1990a: 72s.) se han considerado como una subclase de adverbios y adjetivos utilizados para expresar una valoración subjetiva que hace el enunciador sobre el estado de las cosas, basándose en aspectos emocionales o conectados con el grado de realidad de los hechos, y por tanto relacionados con aspectos modales o emocionales. A su vez, esta categoría se ha subdividido en operadores hipotéticos, evaluativos y emotivos, presentados en los siguientes ejemplos acorde a este orden:

(14) Vielleicht sollten wir unser Haus verkaufen und in ein kleineres ziehen. Rechnet sich das?

(Stern 20/2003. Anunciante: HypoVereinsbank).

(15) Heute bin ich klüger und empfehle meinen Kunden einfach Getränkekartons von Tetra Pak.

(Stern 45/2002. Anunciante: Tetra Pak).

(16) Kompromisslos sind sie noch immer. Und glücklich sowieso.

(Stern 30/2002. Anunciante: Sixt und Matt).

Dentro de los recursos discursivos se ha identificado el llamado "sujeto vivencial" (Wahrnehmungsträger), diferenciándose de la referencia directa a los interlocutores del grupo anterior por referirse el primero a la valoración de la subjetividad en su vertiente relativa a la experiencia y no como indicador del rol de los interlocutores en un diálogo escenificado. Mediante su uso el enunciador manifiesta emociones, sentimientos y sensaciones con los que el destinatario pueda identificarse, y por tanto sentirse más próximo al consumidor evocado del producto o servicio anunciado. Su categorización se ha marcado a partir de la clasificación semántica del verbo y la voz activa y pasiva en referencia a la primera persona, expresada tanto por pronombres personales como por pronombres posesivos. En el ejemplo 17 puede observarse el monólogo interior de un enunciador (wir/uns) con las mismas inquietudes y preocupaciones que el potencial consumidor, que puede acabar empatizando con el producto anunciado (hipotecas bancarias) a través de su identificación con el enunciador escenificado:

(17) Wenn wir zusammen wohnen würden, könnten wir uns eine grössere Wohnung leisten, eine mit Badewanne. Wir könnten zusammen baden. Was passiert, wenn wir uns trennen? Wie würde ich die Miete bezahlen?

(Stern 30/2003. Anunciante: Hypovereinsbank).

También dentro de los recursos discursivos, se ha codificado la apelación a necesidades reales, estrategia mediante la que se activan presupuestos en relación con el producto o servicio relevantes para el destinatario en términos de derechos socialmente reconocidos, y no en relación con el rol social imaginario de un supues- 
to consumidor evocado, así se considera una estrategia de cortesía donde se reconocen las necesidades del destinatario y se actúa según sus intereses. En este punto cabe señalar que las asociaciones son de conocimiento deductivo y procedimental, y permiten crear en consecuencia inferencias más amplias a las propiamente delimitadas al significado semántico-referencial codificado en las piezas léxicas. Como categoría abierta, tras la identificación y codificación en nodos libres de las informaciones semántico-referenciales que apelan a necesidades reales, estas se han agrupado en los siguientes marcos conceptuales de valores compartidos reelaborados a partir de las propuestas mencionadas en el apartado 4 (Dmoch 1997: 20s.; Kroeber-Riel 2000; Tepe / Zur Nieden 2001):

1. Derecho a expresar las emociones.

2. Derecho a la individualidad.

3. Derecho al placer y al tiempo libre.

4. Derecho a la salud y a la conservación del medio ambiente.

5. Derecho a la diferencia cultural y a la reafirmación de valores éticos propios o de una cultura ajena ( $c f$. valores multiculturales e internacionalistas de Kroeber-Riel 2000).

En el ejemplo siguiente (18) se presenta el uso de una autoridad/celebridad para otorgar credibilidad al producto anunciado, en este caso utilizando la figura del fundador de una compañía de éxito (Mathias Stinnes) ligada empresarialmente a una empresa ya reconocida como es la Deutsche Bahn. En este caso, se ha tenido en cuenta la creación de la autoridad a través de estrategias emocionales puesto que tiene el papel de reforzar la credibilidad y otorgar legitimidad al texto publicitario (Van Leeuwen 2007: 94), pudiendo ser representada testimonialmente tanto por un personaje conocido como por un profesional especializado, estadísticas o encuestas, que atribuyen la autoridad tanto al enunciador escenificado como a aquellos elementos que apoyen la estrategia textual:

(18) Im Jahre 1808 legte Mathias Stinnes den Grundstein für eine beispiellose erfolgsgeschichte. Ab September 2003 setzen wir diese geschichte fort. Denn im Zusammenschluss von Stinnes und der Deutschen Bahn verbinden sich zwei starke Partner mit einem einzigartigen Leistungsangebot.

(Stern 21/2002. Anunciante: Stinnes Logistics).

A continuación, en el marco conceptual derecho al placer y al tiempo libre y por tanto contrapuesto al derecho al trabajo, se plantea la necesidad real de descanso (Urlaub) y también de los momentos de ocio en el tiempo libre (Kann ich dann nicht mehr so oft essen gehen?):

(19) Ich brauche Urlaub. Morgen fange ich an zu sparen. Kann ich dann nicht mehr so oft essen gehen?

(Stern 20/2003. Anunciante: Hypovereinsbank). 
Seguidamente se ejemplifica la escena conceptual derecho a la salud y conservación del medio ambiente en coexistencia con la del derecho a la individualidad, identificada la primera a partir de piezas léxicas como Garten, Erholung, Kraft, Energie, Oase o Paradies, y la segunda mediante individuell:

(20) MEIN GARTEN - EIN ORT, AN DEM MEINE LEBENSLUST AUFBLÜHT. Jeder wünscht sich einen Ort, der anregt. An dem Körper und Geist Erholung finden und der Kraft und Energie spendet. Der eigene Garten ist so ein Ort. Er ist eine grüne Oase und ein kleines, privates Paradies. Und er ist genauso individuell wie Sie: $\mathrm{Ob}$ Sie von einem Steingarten träumen, sich einen Schwimmteich wünschen oder Ihre Lieblingsecke mit einer neuen Terrasse verschönern wollen.

(Brigitte 15/2000. Anunciante: Garten \& Landschaft).

En último lugar, en cuanto a la escenificación de necesidades creadas (ejemplo 21), cabe recordar que esta estrategia es un instrumento recurrente en la comunicación publicitaria, donde se apela al rol social imaginario de un supuesto consumidor evocado. Esta categoría comparte con la anterior el mismo ámbito de clasificación, aunque en este caso no se interpreta como una necesidad real del destinatario, sino como una necesidad creada a partir de un target aspiracional, representado para que el destinatario desee emularlo a través de la adquisición del producto o servicio anunciado. Por tanto, partiendo del contraste entre realidad y deseo, este tipo de estrategia apelará a emociones, sentimientos y sensaciones que traten de evocar un mundo ideal al que se puede acceder mediante la compra de ese producto o servicio. Así, en la formulación del anuncio publicitario se proponen unos roles determinados con los que el destinatario pueda identificarse, para más tarde traspasarse a la realidad desde un complejo de signos:

(21) Hotel LTI Villa Sun Island, Malediven.

Seit hier die ersten Entdecker vor Anker gingen, hat sich einiges verändert. Die Palmen sind ein wenig gewachsen.

(Stern 37/2003. Anunciante: Jahn Reisen).

\section{Conclusiones}

Debido a la naturaleza sincrónica del análisis, el objetivo de la investigación, más que constatar un cambio de valores en la comunicación publicitaria, ha sido confirmar o descartar la presencia de estrategias de implicación emocional en los anuncios publicitarios seleccionados ${ }^{6}$. Así, el análisis cualitativo ha permitido poner de manifiesto la presencia e importancia de estas estrategias en la formulación de este tipo textual, tanto mediante recursos lingüísticos como estrategias indirectas. Igualmente, se ha constatado su utilización como herramienta para construir la estrategia del enunciador con el fin de persuadir al destinatario. Estos resultados no hacen sino

${ }^{6}$ Para una corroboración cuantitativa de estos resultados puede consultarse PANADÉs (2013). 
corroborar el rol determinante de la emocionalidad junto al de la transferencia de información, que se enmarca así en la tendencia indiscutible de los últimos años hacia la globalización, donde se describe la sociedad no solamente como de la información o del riesgo, sino también con atributos como postindustrial, vivencial y postmoderna (Brunner 1997: 12ss.), sumándose así a las aportaciones de la llamada inteligencia emocional, donde se establece una relación entre razón y emoción y se promueve la importancia de las emociones en la toma de decisiones humanas.

Por último, cabe destacar la utilidad de esta investigación en el estudio de las lenguas con finalidades específicas, puesto que puede ser explotada didácticamente como material docente en el aula tanto por su contenido como por el tipo de comunicación en el que se enmarca. A este respecto, Cook (1992: 16) ya ha apuntado la idoneidad de este tipo textual en un contexto educativo, puesto que sirve como estímulo para discutir o debatir temas actuales de la sociedad y por ser un tipo de comunicación omnipresente e inevitable actualmente, pero también entretenido y divertido. Cabe mencionar además que se ha pretendido hacer una contribución al estudio del lenguaje publicitario y de la presencia de la emocionalidad en un mundo en constante cambio como es el actual. El lenguaje publicitario, como extensión del lenguaje cotidiano, se configura como termómetro de cambios políticos, económicos, culturales y sociales, por lo que la aparición de métodos innovadores en publicidad podrá considerarse en paralelo a la evolución de la sociedad: "Medien übertragen nicht einfach Botschaften, sondern entfalten eine Wirkkraft, welche die Modalitäten unseres Denkens, Wahrnehmens, Erfahrens, Erinnerns und Kommunizierens prägt" (Krämer 1998: 14).

Como propuesta final pueden plantearse nuevas vías de investigación como realizar un estudio contrastivo con anuncios publicitarios en prensa escrita en español, derivando así en un estudio intercultural entre ambas lenguas, o la realización de estudios similares en función de productos conocidos por el público, donde la información es menor.

\section{Referencias bibliográficas}

ArIELY, D., Las trampas del deseo. Barcelona: Ariel 2008.

Baumgart, M., Die Sprache der Anzeigenwerbung. Eine linguistische Analyse aktueller Werbeslogans. Heidelberg: Physica (Konsum und Verhalten 37) 1992.

Brown, P. / Levinson, S. C., Politeness. Some Universals in Language Usage. Cambridge: Cambridge University Press 1987.

BrunNer, R., «Die Fragmentierung moderner Gesellschaften», en: Becker, B. / PAETAU, M. (eds.), Virtualisierung des Sozialen: die Informationsgesellschaft zwischen Fragmentierung und Globalisierung. Frankfurt am Main/Nueva York: Campus 1997, 11-27.

BussmanN, H., Lexikon der Sprachwissenschaft. Stuttgart: Kröner 1990.

Calsamiglia, H. / Tuson, A., Las cosas del decir. Manual de análisis del discurso. Barcelona: Ariel Lingüística 2004.

CoOK, G., The discourse of advertising. Londres/Nueva York: Routledge 1992.

DAmÁsio, A., El error de Descartes: la emoción, la razón y el cerebro humano. Barcelona: Crítica 2006. 
De Beaugrande, R. A. / Dressler, W.U., Introducción a la lingüística del texto. Barcelona: Ariel 1997.

Dirscherl, K., «Das Fremde als Markenzeichen», en: LenZ, B. / LÜSEBrinK, H. J. (eds.), Fremdheitserfahrung und Fremdheitsdarstellung in okzidentalen Kulturen. Passau: Richard Rothe 1999, 133-144.

Dмосн, T., Interkulturelle Werbung: Verhaltenswissenschaftliche Grundlagen für die Standardisierung erlebnisbetonter Werbung. Aquisgrán: Shaker 1997.

Edell, J. A., «Emotion and Advertising: A Timely Union», en: Agres, S. J. et al. (eds.), Emotion in Advertising: Theoretical and Practical Explorations. Nueva York: Quorum Books 1990, XIII-XVIII.

Eelen, G., A Critique of Politeness Theories. Manchester: Saint Jerome Publishing 2001.

GarCía Velasco, M. J., «Code-breaking/code-making: A new Language Approach in Advertising», Journal of Pragmatics 43/8 (2011), 2.095-2.109.

Gigerenzer, G., Adaptative Thinking: Rationality in the Real World. Nueva York: Oxford University Press 2000.

Hamilton, S., «Informative Advertising in Differenciated Oligopoly Markets», International Journal of Industrial Organization 27 (2009), 60-69.

Helbig, G., Lexikon deutscher Modalwörter. Leipzig: Verlag Enziklopädie 1990a.

Helbig, G., Lexikon deutscher Partikeln. Leipzig: Verlag Enziklopädie 1990b.

HENNECKE, A., Im Osten nichts Neues? Eine pragmalinguistisch-semiotische Analyse ausgewählter Werbeanzeigen für Ostprodukte für den Zeitraum 1993 bis 1998. Frankfurt am Main: Peter Lang 1999.

House, J., «Politeness in Germany: Politeness in Germany?», en: Hickey, L. / STEwART, M. (eds.), Politeness in Europe. Clevedon/Buffalo/Toronto: Multilingual Matters 127 2005, 13-28.

JANICH, N., Fachliche Information und inszenierte Wissenschaft. Tübingen: Gunter Narr 1998.

Janich, N. / Greule, A., Werbesprache. Ein Arbeitsbuch. Tübingen: Narr 2001.

JiMÉNEZ Morales, M., De l'estereotip publicitari adult a la realitat preadolescent. Influència de la publicitat en els trastorns del comportament alimentari en nens $i$ nenes de 8 a 12 anys. [Tesis doctoral]. Barcelona: Universitat Pompeu Fabra 2006. http://www.tdx. cat/handle/10803/7521. [27/11/2013].

KRÄMER, S., «Was haben die Medien, der Computer und die Realität miteinander zu tun?», en: KrÄMER, S. (ed.), Medien, Computer, Realität: Wirklichkeitsvorstellungen und neue Medien. Frankfurt am Main: Suhrkamp 1998, 9-26.

Kroeber-RIEL, W., «Auffallen ist alles - Von Information kann keine Rede sein», en: KaLt, G. (ed.), Öffentlichkeitsarbeit und Werbung. Instrumente, Strategien, Perspektiven. FrankfurtMain: IMK in der Verl.-Gruppe Frankfurter Allgemeine Zeitung 1994, 245-248.

Kroeber-RIEL, W., Strategie und Technik der Werbung: Verhaltenswissenschaftliche Ansätze. Stuttgart: Kohlhammer 2000.

LACroix, M., El culte a l'emoció. Barcelona: La Campana 2005.

LAGERWERF, L. et al., «Processing visual Rethoric in Advertisements: Interpretations Determined by Verbal Anchoring and Visual Structure», Journal of Pragmatics 44/13 (2012), 1.836-1.852.

LaRminauX, C., Traduction-adaptation du discours publicitaire: analyse comparée des sites Internet du groupe Danone, versions pour la France et pour l'Espagne. [Tesis doctoral]. Salamanca: Universidad de Salamanca. 2010. http://gredos.usal.es/jspui/handle/10366/83294. [27/11/2013]. 
LeVelt, W. J. M., Speaking: from Intention to Articulation. Cambridge (Mass.): MIT Press 1989.

LeVinson, S. C., Pragmatik. Tübingen: Niemeyer 2000.

Messaris, P., Visual Persuasion. The Role of Images in Advertising. Thousand Oaks: SAGE Publications 1997.

MöHn, D., «Textsorten und Wissentransfer», en: Brinker, K. et al. (eds.), Text- und Gesprächslinguistik. Linguistics of Text and Conversation. Ein internationales Handbuch zeitgenössischer Forschung. H.S.K. VI, 16.1, 51. Berlín/Nueva York: de Gruyter 2000, 561-574.

Moser, K., Werbepsychologie. Göttingen: Hogrefe 2002.

NöTH, W., «Der Zusammenhang von Text und Bild. Textkonstitution IV: Textproduktion Textgestaltung - Textrezeption», en: BRINKER, K. et al. (eds.), Text- und Gesprächslinguistik. Linguistics of Text and Conversation. Ein internationales Handbuch zeitgenössischer Forschung. H.S.K. VI, 16.1, 46. Berlín/Nueva York: de Gruyter 2000, 489-496.

PANADÉs, M., Estrategias persuasivas en los anuncios de prensa escrita alemana: desarrollo temático, ilocución e implicación emocional. [Tesis doctoral]. Barcelona: Universitat de Barcelona 2013. http://www.tdx.cat/handle/10803/110574. [27/11/2013].

PeÑa Cervel, S., Topology and Cognition. Múnich: Lincom Europa 2003.

PUIG I Borràs, N., Emocions i publicitat. El lligam emocional entre l'emissor i el receptor de la publicitat. [Tesis doctoral]. Girona: Universitat de Girona 2010. http://www.tdx. cat/handle/10803/31846. [27/11/2013].

Rentel, N., «Die Didaktisierung von Phraseologismen im DaF-Unterricht anhand mulimodaler Texte», Linguistik online 47/3 (2011). http://www.linguistik-online.de/47_11 rentel.html. [27/11/2013].

RöMER, R., Die Sprache der Anzeigenwerbung. Düsseldorf: Schwann 1968.

Schmitt, D., Die Sprache der Werbung. Methoden der Textanalyse. Múnich: GRIN 2008.

Schwitalla, J., «Beteiligungsrollen im Gespräch», en: BurkHARDT, A. et al. (eds.), Gesprochene Sprache. Forschung und ihre Entwicklung zu einer Gesprächsanalyse. Berlín/Nueva York: de Gruyter 2001, 1355-1361.

Siebold, K., Actos de habla y cortesía verbal en español y en alemán. Estudio pragmalingüistico e intercultural. Studien zur romanischen Sprachwissenschaft und interkulturellen Kommunikation. Frankfurt am Main: Peter Lang 2000.

SowINSKI, B., Werbung. Tübingen: Niemeyer 1998.

STÖскL, H., Die Sprache im Bild - Das Bild in der Sprache. Berlín: de Gruyter 2004.

TePe, P. / Zur Nieden, B., Mythos \& Literatur. Würzburg: Königshausen \& Neumann 2001.

TIEnKen, S., Alltagsgattungen und der Ort von Kultur. Sprachwissenschaftliche und kulturanalytische Studien anhand von Milchverpackungen in Deutschland und Schweden. [Tesis doctoral]. Estocolmo: Stockholm University 2008. http://urn.kb.se/resolve?urn= urn:nbn:se:su:diva-8248. [27/11/2013].

Ungerer, F., «Ads as News Stories, News Stories as Ads: The Interaction of Advertisements and Editorial Texts in Newspapers», Text 24 (3) (2004), 307-328.

Van Leeuwen, T., «Legitimation in Discourse and Communication», Discourse and Communication 1 (1) (2007), 91-112. http: //dcm. sagepub. com/cgi/content/ abstract/1/ /1/91. [27/11/2013].

WeHNER, C., Überzeugungsstrategien in der Werbung. Eine Längsschnittanalyse von Zeitschriftenanzeigen des 20. Jahrhunderts. Opladen: Westdeutscher 1996.

WierzBickA, A., Primes and Universals. Oxford: Oxford University Press 1996. 